\title{
SYNCHRONIZATION APPROACH TO ANALYSIS OF BIOLOGICAL SYSTEMS
}

\author{
MICHAEL G. ROSENBLUM, ARKADY S. PIKOVSKY and JÜRGEN KURTHS \\ Department of Physics, Potsdam University \\ Am Neuen Palais, 10, D-14415, Potsdam, Germany
}

Received 3 December 2003

Revised 2 February 2004

Accepted 4 March 2004

\begin{abstract}
In this article we review the application of the synchronization theory to the analysis of multivariate biological signals. We address the problem of phase estimation from data and detection and quantification of weak interaction, as well as quantification of the direction of coupling. We discuss the potentials as well as limitations and misinterpretations of the approach.
\end{abstract}

Keywords: Synchronization; multivariate data; interaction strength; directionality.

\section{Introduction}

Synchronization is a fundamental nonlinear phenomenon, and it plays an important role in various fields of science and engineering [1-5]. Very often it is found in live systems, being observed on a level of single cells, physiological subsystems, organisms and even on the level of populations [3,6,7]. Sometimes, this phenomenon is essential for a normal functioning of a system, e.g. for a coordinated motion of several limbs or for the performance of a pacemaker, where many cells fire synchronously, and in this way produce a macroscopic rhythm that governs respiration, heart contraction, etc. Sometimes, the onset of synchrony leads to a severe pathology, e.g. in case of the Parkinson's disease, when locking of many neurons results in the tremor activity. Quite often, the functional role of synchrony is yet unknown, e.g. in case of cardiorespiratory coordination [8-12] or in case of mutual entrainment of respiration and locomotion; possibly its appearance is just a manifestation of a general property of self-sustained oscillators - to adjust their rhythms due to a weak interaction. On the other hand, onset or cessation of synchrony reflects variation in the state of the complex system, and therefore may provide important physiological information.

In this paper we review the application of synchronization ideas to analysis of multivariate data measured from biological systems. We discuss and compare 
traditional and recently introduced techniques aimed to reveal and quantify the interaction between physiological subsystems from measurements. The main idea is to consider the inverse problem, i.e. to analyze the observed phase relations in order to obtain some information on the interaction of the systems generating the signals. In particular, we are interested in quantification of the strength and of the interaction and estimation of the directionality of coupling. We pay especial attention to the discussion of possible pitfalls and limitations of the method.

We note that such well-known effects as classical and stochastic resonance $[13,14]$ in some respects are close to synchronization and cannot be distinguished from it within the framework of a passive experiment, i.e. when the system is simply observed, without any controlled variation of its parameters (for a discussion of active and passive experiments see [3]). A common feature of these effects is an appearance of a relation between phases and frequencies of two or many signals, and therefore the analysis of these relations can be used in case of a resonance as well. In particular, this approach has been successfully exploited for the investigation of stochastic systems $[5,15-17]$.

\section{Estimation of Instantaneous Phases}

Prior to the analysis of phase relations we have to estimate phases from data. There exist three main approaches to the problem. One is based on the construction of the complex analytic signal $\zeta(t)[18]$ from a scalar experimental time series $s(t)$ via the Hilbert transform (HT)

$$
\zeta(t)=s(t)+i s_{H}(t)=A(t) e^{i \phi(t)}, \quad s_{H}(t)=\pi^{-1} \mathrm{P} . \mathrm{V} . \int_{-\infty}^{\infty} \frac{s(\tau)}{t-\tau} d \tau,
$$

where $s_{H}(t)$ is HT of $s(t)$. Equation (1) unambiguously provides an instantaneous phase $\phi(t)$ and an amplitude $A(t)$. Note that HT is parameter free. Practical hints for computation and usage of the HT, as well as further citations can be found in $[3,19]$. Here we briefly mention the crucial points:

- Mathematically, HT is defined for an arbitrary signal. However, $\phi(t)$ and $A(t)$ admit a clear physical interpretation only for narrow band signals. If the signal has no well-expressed peak in its power spectrum, then the computation of the phase and application of the synchronization approach is highly doubtful.

- A complex signal that can be considered as a mixture of several narrow band processes should be first decomposed into oscillatory components that can be considered as signals with slowly varying amplitude and frequency; as the next step, the phases of these components can be obtained via HT. (Note that sometimes it is difficult to decide whether a peak in the spectrum represents another process or a harmonic.) Decomposition can be done by means of a band-pass filter or by more sophisticated techniques like independent component analysis.

- Determination of $\phi(t)$ is very sensitive to low-frequency trends, what makes the preprocessing of the data a crucial step in the analysis. 
The second approach exploits the wavelet analysis with a complex wavelet function and provides a phase (and an amplitude) as functions of time for a certain spectral frequency band $[20,21]$ :

$$
A(t ; f) e^{\imath \phi(t ; f)}=\int_{-\infty}^{\infty} s(\tau) \Psi^{*}(t, \tau ; f) d \tau,
$$

where $\Psi(t, \tau ; f)$ is the Morlet, or Gabor, wavelet:

$$
\Psi(t, \tau ; f)=\sqrt{f} \exp (\imath \cdot 2 \pi f(\tau-t)) \exp \left(-\frac{(\tau-t)^{2}}{2 \sigma^{2}}\right) .
$$

This procedure is equivalent to a band-pass filtration and subsequent HT of the signal $s(t)$ [22]. The central frequency of the filter is $f$, and its width is determined by the parameter $\sigma$.

Third, the phase can be very easily introduced for processes that can be treated as a series of well-defined events taking place at times $t_{k}$ (point processes). Examples include signals characterizing heart beat or neuron firing [23]. If the interval between two events can be considered as a cycle, then it is natural to say that the phase increment between the events is exactly $2 \pi$. Hence, we can assign to the times $t_{k}$ the values of phase $\phi\left(t_{k}\right)=2 \pi k$, and for arbitrary instant of time $t_{k}<t<t_{k+1}$ take

$$
\phi(t)=2 \pi k+2 \pi \frac{t-t_{k}}{t_{k+1}-t_{k}} .
$$

Finally, we mention that definition and practical determination of a phase of a complex signal in the context of the synchronization analysis remains an open problem. One approach, called locking-based frequency measurement was suggested in [24]. The idea of this approach is to use the signal under study to drive a set of uncoupled limit cycle oscillators with different natural frequencies. A subset of these probe oscillators can be entrained by the common forcing, and therefore synchronize in between; the frequency and the phase of these locked oscillators can be taken as an estimate of the frequency and phase of the original signal.

\section{Quantification and Interpretation of Phase Relations}

As soon as the phases of two signals are estimated by appropriate methods, the relations between phases can be quantified. For this purpose we recall that the interaction of noisy oscillators is reflected in the distribution of the cyclic relative phase

$$
\psi_{n, m}(t)=\left(n \phi_{1}(t)-m \phi_{2}(t)\right) \bmod 2 \pi,
$$

where $n, m$ are integers characterizing the order of locking (see, e.g. [3]). For uncoupled oscillators the distribution of $\psi_{n, m}$ is uniform, whereas the interaction makes this distribution unimodal. Hence, the strength of interaction between the systems can be estimated via quantification of the form of the distribution of $\psi_{n, m}$. The correspondent measures are called synchronization indices; usually they are normalized in such a way that zero index corresponds to complete independence, whereas unit index corresponds to perfect phase locking. An index can be based on the Shannon 
entropy of the distribution of $\psi_{n, m}(t)$ [25]. Alternatively, it can be taken equal to the intensity of the first Fourier mode of the distribution $[19,20]$ :

$$
\rho_{n, m}^{2}=\left\langle\cos \psi_{n, m}\right\rangle^{2}+\left\langle\sin \psi_{n, m}\right\rangle^{2} .
$$

The advantage of this measure is the absence of parameters. Note that the integers $n, m$ can be evaluated from the estimation of the average frequencies of two signals (e.g. from their power spectra), or found by trial and error by looking for the maximum of $\rho_{n, m}$.

If one of the signals can be considered as a point (event) process, then the phase interrelation can be conveniently quantified with the help of the phase stroboscope. For example, this technique turned out to be useful in the analysis of cardiorespiratory interaction $[3,10,19]$. Phase stroboscope implies, that the second signal is observed at the moments when the events in the first signal occur, i.e. when $\phi_{1}(t)=2 \pi n$. So, in case of the cardiorespiratory data, the phase of the respiratory signal is determined at the instants of the appearance of the R-peak in the cardiogram. Interaction between the systems can be revealed by means of a graphical tool, called synchrogram, and quantified by means of characterization of the stroboscopically observed phase [11].

Concerning the interpretation of the phase relations we want to emphasize the difference between the approach developed in our previous papers (see [19] for a review) and the approach, suggested in $[20,21]$. Our approach, based on the theory of coupled oscillators assumes that there exists a unique phase for each of the interacting oscillators. Therefore the preprocessing, e.g. by means of a band-pass filter, is aimed to extraction of a certain signal from its mixture with other signals and broad band noise. This extracted signal is assumed to be generated by one of the subsystems under study, and the phase of this subsystem is estimated and $n: m$ relation between phases is quantified. On the contrary, in the approach of $[20,21]$ the phase is computed subsequently for all spectral frequency bands by means of the continuous variation of the wavelet parameter corresponding to the central frequency of the band-pass filter. Then, a $1: 1$ phase relation of two signals is quantified for each frequency band, providing the index $\rho_{1,1}$ as a function of the spectral frequency $f . \rho_{1,1}(f)$ can be considered in analogy to the classical tool of the cross-spectrum analysis, namely to the coherence function $\gamma(f)$. Similarly to $\gamma(f)$, $\rho_{1,1}(f)$ characterizes the interdependence between certain frequency components of two signals; however, in contrast to $\gamma(f), \rho_{1,1}(f)$ takes into account only the phase information. Thus, the technique $[20,21]$ should be considered as an extension of the cross-spectrum analysis rather than in the framework of coupled oscillator approach.

Concluding this section, we comment on the relevance of possible relations between the amplitudes of the signals. Our approach is motivated by the synchronization phenomenon, where the phase (and frequency) relations are of primary importance, whereas the amplitudes are generally not much influenced by a weak interaction, and, e.g. in case of chaotic systems can be weakly correlated. However, the experimental data do not always comply with this model, and then the amplitude relations can be also important. An example is given in [26], where interhemispheric synchrony of spontaneous beta oscillations in humans was analyzed. 
It was shown that the phase relations are amplitude-dependent, namely, the synchronization index increases with the amplitude of signals; it was hypothesized that this is due to the increase of the signal-to-noise ratio. However, a visual inspection of the signals shows a strong amplitude modulation of signals (that is why these oscillations are called spontaneous) which is typical for narrow band noise. So, we may assume that the systems under study cannot be considered as self-sustained oscillators, and, therefore, the notion of synchronization may not be applicable in this case. The observed phase relation should be rather interpreted in terms of a resonance, where the amplitude relations, certainly, are important.

\section{Asymmetric Phase Relations}

If an interaction between two oscillators is established, the next step in the analysis can be the quantification of the direction of coupling. This question is nontrivial because synchronization may be caused either by biderectional or by unidirectional coupling. We outline here two approaches. One, based on information theory, quantifies the direction of the information flow [27]; it operates with raw signals. This approach goes back to the mutual predictability concept of Granger [28] that we can briefly formulate as follows: if system 2 acts on system 1 , then we can better predict the future of the system 1 if we take into account the information on the system 2; this can be also formulated in terms of entropy. The other approach [29] works with the phase data and is based on the coupled oscillator theory.

The main idea is that in case of weak coupling the description of two interacting oscillators can be reduced to the phase dynamics

$$
\dot{\phi}_{1,2}=\omega_{1,2}+f_{1,2}\left(\phi_{2,1}, \phi_{1,2}\right)+\xi_{1,2},
$$

where $\phi_{1,2}$ are phases, $\omega_{1,2}$ are natural frequencies, and the terms $f_{1,2}$ describe the coupling between the systems. The random terms $\xi_{1,2}$ describe noisy perturbation and/or chaotic amplitude dynamics. Given the time series of phases, $\phi_{1,2}\left(t_{k}\right)$, we consider the phase increments during some fixed time $\tau, \Delta_{1,2}(k)=\phi_{2,1}\left(t_{k}+\tau\right)-$ $\phi\left(t_{k}\right)$. These easily obtained new time series can be considered as generated by some unknown two-dimensional noisy map

$$
\Delta_{1,2}=\omega_{1,2} \tau+\mathcal{F}_{1,2}\left(\phi_{2,1}, \phi_{1,2}\right)+\eta_{1,2} .
$$

Deterministic parts of this map can be estimated by fitting the dependencies of $\Delta_{1}$ and $\Delta_{2}$ on $\phi_{1}, \phi_{2}$. As the seeked functions $\mathcal{F}$ are $2 \pi$-periodic with respect to both arguments, the natural choice of the probe function is the Fourier-series.

As soon as the map (5) is identified, the information on the directionality is provided by the coefficients of two Fourier series approximating the unknown functions $\mathcal{F}_{1,2}$. However, this information is not easy to interpret. Here we face the problem how to quantify the direction of coupling by one number, what is definitely ambiguous. One way to introduce such a number, called directionality index, was suggested in [29]. Let $F_{1,2}$ be the fits to $\mathcal{F}_{1,2}$. The sensitivity of the phase dynamics of one system with respect to the variation of the phase of the second system can be quantified by the average of the derivative $\frac{\partial F_{1,2}}{\partial \phi_{2,1}}$. Namely, we characterize this 
by computing the coefficients

$$
c_{1,2}^{2}=\left\langle\frac{\partial F_{1,2}}{\partial \phi_{2,1}}\right\rangle
$$

where \langle\rangle stays for averaging over $\phi_{1,2}$. Finally, the directionality index is computed as

$$
d^{(1,2)}=\frac{c_{2}-c_{1}}{c_{1}+c_{2}}
$$

This index varies from 1 in the case of unidirectional coupling $1 \rightarrow 2$ to -1 in the opposite case, $2 \rightarrow 1$. Intermediate values correspond to bidirectional coupling, and the case $d^{(1,2)}=0$ can be denoted as symmetric coupling. Note, however, that except for unidirectional driving, the definition of directionality is ambiguous.

We note also that the predictability (information-theoretical) approach can be also applied to the phase data $[30,31]$. For examples of application to the cardiorespiratory and brain data see $[30,32]$. Statistical properties of the directionality estimation are considered in [33].

\section{Discussion: Potentials, Limitations and Pitfalls}

Synchronization analysis proved to be a useful tool in experimental investigation of different biological systems. So, tendency to phase locking of different order $n: m$ was revealed in a group of young athletes [10,34] and in normal adults [12]. Strength and directionality of the cardiorespiratory interaction in healthy newborns was studied in $[11,30,35]$ in dependence on the age and sleep stages, what revealed the dependence of the interaction parameters on the respiratory frequency and provided the information on the role of the kinetics involved in the vagal-atrial transmission. As further examples we mention the prediction and localization of epileptic activity [36,37], analysis of the role of interbrain synchronization and localization of pathological brain activity in Parkinson's disease [25,38], or nephron-nephron interaction [39]. Stochastic phase synchronization in sensory systems and its role in mediating sensory responses was studied in a series of papers of the St.Louis group, in experiments with electrosensitive afferent neurons of the paddlefish and with light-sensitive and mechanosensitive neurons of the crayfish, as well as theoretically $[15-17,23,40]$. The algorithm for estimation of the directionality in coupling was exploited for understanding of the functional connectivity in the brain during a prescribed motor task $[32,41]$.

In what follows we discuss the limitations of this synchronization approach and problems of an interpretation of the results. First of all we emphasize again that blind application of the Hilbert transform to a broad band signal can provide physically meaningless results. The first step of the synchronization analysis - estimation of instantaneous phases requires a careful choice of the preprocessing and is crucial.

Next, we stress that the detection of phase relations between two signals does not always mean that we deal with a synchronization process. These relations can be, e.g. a manifestation of classical or stochastic resonance. Quantification and analysis of time dependencies of such relations can nevertheless provide useful information on the biological system, though one should be careful in the conclusions regarding underlying physical mechanisms. 
The synchronization approach is based on the assumptions that we deal with interacting self-sustained oscillators, and that the signals we measure represent the dynamics of different systems. Violation of these assumptions, e.g. when two signals reflect different variables of the same system or are input and output of some passive transformer, makes the analysis hardly useful.

Finally, we mention that in the physiological literature, in particular in neuroscience, the term "synchrony (synchronization)" is very often used as a synonym to "correlation". Hence, the cross-spectral (cross-correlation) technique is used as a standard tool to quantify synchrony. If we understand synchronization in a physical sense, i.e. as an adjustment of phases and frequencies of interacting oscillating objects, then it is clear that this tool is not an appropriate one. Indeed, high coherence in the spectral domain can be a result of other types of interaction than weak synchronizing coupling, e.g. it can be observed in case of modulation. Moreover, the cross-spectral (cross-correlation) technique accounts for both amplitude and phase interrelations. Generally, correlation (coherence) and synchronization analysis address different aspects of interaction and can, e.g. lead to different results in localization of pathological brain activity $[19,25]$. Next important point is that sometimes the conclusion on the driver-response relationship (i.e. on the directionality) is made on the basis of the phase of the cross-spectrum at a certain dominating frequency. It is concluded that the system that lead in phase is the driver. This concept, inherited from the input-output approach to passive transformers, definitely fails for coupled oscillators, where the phase shift in the synchronous regime depends on coupling functions and natural frequencies of both interacting systems, and these factors cannot be separated. [Indeed, considering the simplest model of two interacting oscillators, $\phi_{1,2}=\omega_{1,2}+\varepsilon_{1,2} \sin \left(\phi_{2,1}-\phi_{1,2}\right)$ (Adler equations, cf. with Eqs. (4)), one easily obtains for the phase difference $\phi_{1}-\phi_{2}=\arcsin \left(\left(\omega_{1}-\omega_{2}\right) /\left(\varepsilon_{1}+\varepsilon_{2}\right)\right)$.] The same is valid for the estimation of the delays by re-computation of the phase shift (obtained from the cross-spectrum) into conduction time (delay): justified in the treatment of passive systems, this approach leads to spurious results if two signals represent coupled oscillators.

As the last remark, we mention that different notions of synchronization, such as generalized and event synchronization are also used in the analysis of interdependencies from multivariate data $[42-46]$.

\section{Acknowledgements}

In our research on synchronization analysis of biological systems we have been highly inspired by fruitful discussions with Professor Frank Moss. We gratefully dedicate this paper to his 70th birthday.

\section{References}

[1] I. I. Blekhman, Synchronization in Science and Technology, Nauka, Moscow (1981), (In Russian); English translation: ASME Press, New York (1988).

[2] P. S. Landa, Nonlinear Oscillations and Waves in Dynamical Systems, Kluwer Academic Publishers, Dordrecht-Boston-London (1996).

[3] A. Pikovsky, M. Rosenblum and J. Kurths, Synchronization. A Universal Concept in Nonlinear Sciences, Cambridge University Press, Cambridge (2001). 
[4] E. Mosekilde, Yu. Maistrenko and D. Postnov, Chaotic Synchronization. Applications To Living Systems, World Scientific, Singapore (2002).

[5] V. Anishchenko, A. Neiman, V. Astakhov, T. Vadiavasova and L. Schimansky-Geier, Chaotic and Stochastic Processes in Dynamic Systems, Springer Verlag, Berlin (2002).

[6] L. Glass and M. C. Mackey, From Clocks to Chaos: The Rhythms of Life, Princeton Univ. Press, Princeton, NJ (1988).

[7] L. Glass, Synchronization and rhythmic processes in physiology, Nature 410 (2001) $277-284$.

[8] H. Pessenhofer and T. Kenner, Zur Methodik der kontinuierlichen Bestimmung der Phasenbeziehung zwischen Herzschlag und Atmung, Pflügers Archiv 355 (1975) 77-83.

[9] T. Kenner, H. Pessenhofer and G. Schwaberger, Method for the analysis of the entrainment between heart rate and ventilation rate, Pflügers Arch. 363 (1976) 263-265.

[10] C. Schäfer, M. G. Rosenblum, J. Kurths and H.-H. Abel, Heartbeat synchronized with ventilation, Nature 392 (1998) 239-240.

[11] R. Mrowka, A. Patzak and M. G. Rosenblum, Qantitative analysis of cardiorespiratory synchronization in infants, Int. J. of Bifurcation and Chaos 10 (2000) 2479-2488.

[12] M. Bračič and A. Stefanovska, Synchronization and modulation in the human cardiorespiratory system, Physica A 283 (2000) 451-461.

[13] K. Wiesenfeld and F. Moss, Stochastic resonance: From ice ages to crayfish and SQUIDs, Nature 373 (1995) 33-36.

[14] F. Moss, D. Pierson and D. O'Gorman, Stochastic resonance: Tutorial and update, Int. J. of Bifurcation and Chaos 4 (1994) 1383-1397.

[15] A. Neiman, X. Pei, D. F. Russell, W. Wojtenek, L. Wilkens, F. Moss, H. A. Braun, M. T. Huber and K. Voigt, Synchronization of the noisy electrosensitive cells in the paddlefish, Phys. Rev. Lett. 82 (1999) 660-663.

[16] A. Neiman, L. Schimansky-Geier, A. Cornell-Bell and F. Moss, Noise-enhanced phase synchronization in excitable media, Phys. Rev. Lett. 83 (1999) 4896-4899.

[17] A. B. Neiman and D. F. Russell, Synchronization of noise-induced bursts in noncoupled sensory neurons, Phys. Rev. Lett. 88 (2002) 138103.

[18] D. Gabor, Theory of communication, J. IEE London 93 (1946) 429-457.

[19] M. G. Rosenblum, A. S. Pikovsky, J. Kurths, C. Schäfer and P. A. Tass, Phase synchronization: From theory to data analysis, in Neuro-informatics and Neural Modeling, volume 4 of Handbook of Biological Physics, ed. F. Moss and S. Gielen, Elsevier, Amsterdam (2001) 279-321.

[20] E. Rodriguez, N. George, J.-P. Lachaux, J. Martinerie, B. Renault and F. J. Varela, Perception's shadow: Long distance synchronization of human brain activity, Nature 397 (1999) 430-433.

[21] J.-P. Lachaux, E. Rodriguez, M. Le van Quyen, A. Lutz, J. Martinerie and F. J. Varela, Studying single-trials of phase synchronous activity in the brain, Int. J. Bifurc. Chaos 10 (2000) 2429-2440.

[22] R. Quian Quiroga, A. Kraskov, T. Kreuz and P. Grassberger, Performance of different synchronization measures in real data: A case study on electroencephalographic signals, Phys. Rev. E 65 (2002) 041903.

[23] S. Bahar, A. Neiman, L. A. Wilkens and Frank Moss, Phase synchronization and stochastic resonance effects in the crayfish caudal photoreceptor, Phys. Rev. E 65 (2002) 050901.

[24] M. Rosenblum, A. Pikovsky, J. Kurths, G. Osipov, I. Kiss and J. Hudson, Lockingbased frequency measurement and synchronization of chaotic oscillators with complex dynamics, Phys. Rev. Lett. 89 (2002) 264102. 
[25] P. Tass, M. G. Rosenblum, J. Weule, J. Kurths, A. S. Pikovsky, J. Volkmann, A. Schnitzler and H.-J. Freund, Detection of $n: m$ phase locking from noisy data: Application to magnetoencephalography, Phys. Rev. Lett. 81 (1998) 3291-3294.

[26] V. V. Nikouline, K. Linkenkaer-Hansen, J. Huttunen and R. J. Ilmoniemi, Interhemispheric phase synchrony and amplitude correlation of spontaneous beta oscillations in human subjects: A magnetoencephalographic study, NeuroReport 12 (2001) 2487-2491.

[27] T. Schreiber, Measuring information transfer, Phys. Rev. Lett. 85 (2000) 461-464.

[28] C. W. J. Granger, Investigating causal relations by econometric models and cross spectral models, Econometrica 37 (1969) 424-438.

[29] M. Rosenblum and A. Pikovsky, Detecting direction of coupling in interacting oscillators, Phys. Rev. E 64 (2001) 045202(R).

[30] M. G. Rosenblum, L. Cimponeriu, A. Bezerianos, A. Patzak and R. Mrowka, Identification of coupling direction: Application to cardiorespiratory interaction, Phys. Rev. E 65 (2002) 041909.

[31] M. Paluŝ and A. Stefanovska, Direction of coupling from phases of interacting oscillators: An information-theoretic approach, Phys. Rev. E 67 (2003) 055201.

[32] L. Cimponeriu, M. G. Rosenblum, T. Fieseler, J. Dammers, M. Schiek, M. Majtanik, P. Morosan, A. Bezerianos and P. A. Tass, Asymmetric relations between interacting neuronal oscillators, Progress of Theoretical Physics Suppl. 150 (2003) 22-36.

[33] D. A. Smirnov and B. P. Bezruchko, Estimation of interaction strength and direction from short and noisy time series, Phys. Rev. E. 68 (2003) 046209.

[34] C. Schäfer, M. G. Rosenblum, H.-H. Abel and J. Kurths, Synchronization in the human cardiorespiratory system, Physical Review E 60 (1999) 857-870.

[35] R. Mrowka, L. Cimponeriu, A. Patzak and M. G. Rosenblum, Directionality of coupling of physiological subsystems - age related changes of cardiorespiratory interaction during different sleep stages in babies, American J. of Physiology (Regul Comp Integr Physiol) 285 (2003) R1395-R1401.

[36] F. Mormann, K. Lehnertz, P. David and C. E. Elger, Mean phase coherence as a measure for phase synchronization and its application to the EEG of epilepsy patients, Physica D 144 (2000) 358-369.

[37] F. Mormann, R. G. Andrzejak, T. Kreuz, Ch. Rieke, P. David, Ch. E. Elger and K. Lehnertz, Automated detection of a preseizure state based on a decrease in synchronization in intracranial electroencephalogram recordings from epilepsy patients, Phys. Rev. E 67 (2003) 021912.

[38] P. A. Tass, T. Fieseler, J. Dammers, K. Dolan, P. Morosan, M. Majtanik, F. Boers, A. Muren, K. Zilles and G. R. Fink, Synchronization tomography: A method for threedimensional localization of phase synchronized neuronal populations in the human brain using magnetoencephalography, Phys. Rev. Lett. 90 (2003).

[39] N.-H. Holstein-Rathlou, K.-P. Yip, O. V. Sosnovtseva and E. Mosekilde. Synchronization phenomena in nephron-nephron interaction, Chaos 11 (2001) 417-426.

[40] S. Bahar and F. Moss, Stochastic phase synchronization in the crayfish mechanoreceptor/photoreceptor system, Chaos 13 (2003) 138-144.

[41] J. Gross, L. Timmermann, J. Kujala, M. Dirks, F. Schmitz, R. Salmelin and A. Schnitzler, The basis of intermittent motor control in humans, Proc. Nat. Acad. Sci. 99 (2002) 2299-2302.

[42] S. J. Schiff, P. So, T. Chang, R. E. Burke and T. Sauer, Detecting dynamical interdependence and generalized synchrony through mutual prediction in a neural ensemble, Phys. Rev. E 54 (1996) 6708-6724. 
[43] M. Le van Quyen, C. Adam, M. Baulac, J. Martinerie and F. J. Varela, Nonlinear interdependencies of EEG signals in human intracranially recorded temporal lobe seizures, Brain Research 792 (1998) 24-40.

[44] J. Arnhold, P. Grassberger, K. Lehnertz and C. E. Elger, A robust method for detecting interdependences: Application to intracranially recorded EEG, Physica D 134 (1999) 419-430.

[45] R. Quian Quiroga, J. Arnhold and P. Grassberger, Learning driver-response relationships from synchronization patterns, Phys. Rev. E 61 (2000) 5142-5148.

[46] R. Quian Quiroga, T. Kreuz and P. Grassberger, Event synchronization: A simple and fast method to measure synchronicity and time delay patterns, Phys. Rev. E 66 (2002) 041904. 